\title{
Causes of Death in Autopsied Patients with Rheumatoid Arthritis in Japan
}

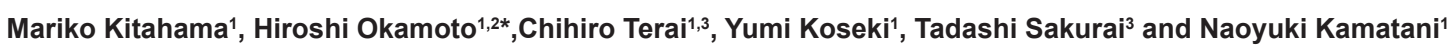

'Institute of Rheumatology, Tokyo Women's Medical University, Tokyo, Japan

${ }^{2}$ Minami-Otsuka Institute of Technology, Minami-Otsuka Clinic, Tokyo, Japan

${ }^{3}$ Saitama Medical Center, Jichi Medical University, Saitama, Japan

\begin{abstract}
Objective: To investigate causes of death in Japanese patients with Rheumatoid Arthritis (RA) and whether recent RA treatment has been associated with a decrease in AA-amyloidosis among RA patients.

Methods: Data from records of RA patients autopsied in Japan during 2000-2004 and 1985-1989 were analyzed. Cause of death, age at death, gender, and incidence of amyloidosis were assessed.

Results: The average age at death in the recent RA group was 69.1 years in 817 females and 70.1 years in 408 males. Unlike the general population, RA females died earlier than males. In the past 15 years, a survival rate of RA patients has improved. The leading cause of death of recently diagnosed RA patients was infection (32.8\%), respiratory diseases $(20.2 \%)$, and AA-amyloidosis (11.3\%). In comparison with patients surveyed in 1985-1989 this represents an increase in deaths from infection and respiratory diseases, a decrease in deaths from RA itself and renal diseases, and no change in deaths from AA-amyloidosis. In RA, more males died of interstitial lung disease (odds 2.94) and more females died from AA-amyloidosis (odds 3.52).
\end{abstract}

Conclusion: Cause of death is significantly different in RA patients compared to the general population. Although outcomes of RA patients have been improving, the incidence of AA-amyloidosis remains high in Japan.

Keywords: Rheumatoid arthritis; Inflammation; Pathology; Malignancies

\section{Introduction}

Numerous studies in the US and Western countries have demonstrated that excessive mortality associated with Rheumatoid Arthritis (RA) is mainly related to cardiovascular death $[1,2]$. Since most studies of mortality due to RA from Japan are relatively small, it is not clear whether cardiovascular death is common in Japanese with RA $[3,4]$.

AA-amyloidosis is caused by sustained elevation of serum amyloid A and prolonged inflammation. Although AA-amyloidosis used to be a major cause of death in patients with RA, the incidence of this complication seems to have declined recently $[4,5]$. Widespread use of Methotrexate (MTX) and the appearance of biologics have changed the treatment of RA. Consequently, complete control of inflammation in RA is becoming more realistic, thereby affecting mortality, causes of death, and complications (e.g., amyloidosis) in RA patients [6,7]. In support of this hypothesis, recent reports have shown improved survival in RA patients treated with MTX or biologics [8].

Diagnosis of AA-amyloidosis requires histological demonstration of AA-amyloid deposition. However, this requirement could lead to an underestimation of AA-amyloidosis among RA population since most studies on RA mortality used causes of death on the death certificate to identify death cause. Toyoshima et al. [9] reported the causes of death in autopsied Japanese RA patients during 1980's and found increased mortality by infection, respiratory diseases and AA-amyloidosis secondary to RA. In this study, we investigated the causes of death in patients with RA at death (RA patients) autopsied during 2000-2004 in Japan and compared our results to the previous ones. By using data from autopsied patients throughout Japan, mortality due to RA could be studied on a large scale and from a pathological point of view.

\section{Materials and Methods}

\section{Data source}

The "Annual of the Pathological Autopsy Cases in Japan" is published by the Japanese Society of Pathology every year and contains records of almost all autopsies performed in Japan by the members of the Japanese Society of Pathology. Data include age at death, gender, clinical diagnoses, and pathological diagnoses of each case. Postmortem examinations were carried out by specialists of pathology approved by the Japanese Society of Pathology, following standard processes of the society. These standard processes have been made in reference to WHO rules for assessing underlying cause of death. We extracted all data from records of patients clinically or pathologically diagnosed with RA during the 2000-2004 time periods. No information was available on whether the RA in each case fulfilled revised American College of Rheumatology (ACR) criteria for RA and what treatment for RA was received [10]. This study was approved by the Institutional Review Board of Tokyo Women's Medical University.

\section{Causes of death}

Causes of death included RA itself, AA-amyloidosis, respiratory disease (interstitial lung disease [ILD] and others), infection

*Corresponding author: Hiroshi Okamoto, Minami-Otsuka Institute of Technology, Minami-Otsuka Clinic, 2-41-9 Minami-Otsuka, Toshima-ku, Tokyo 170-0005 Japan, Tel: +81-(3)-3943-7277; Fax:+81-(3)-3943-9018; E-mail: hokamoto@dream.bbexcite.jp

Received November 15, 2013; Accepted December 20, 2013; Published December 27, 2013

Citation: Kitahama M, Okamoto H, Terai C, Koseki Y, Sakurai T, et al. (2013) Causes of Death in Autopsied Patients with Rheumatoid Arthritis in Japan. J Arthritis 2: 115. doi:10.4172/2167-7921.1000115

Copyright: (c 2013 Kitahama M, et al. This is an open-access article distributed under the terms of the Creative Commons Attribution License, which permits unrestricted use, distribution, and reproduction in any medium, provided the original author and source are credited. 
Citation: Kitahama M, Okamoto H, Terai C, Koseki Y, Sakurai T, et al. (2013) Causes of Death in Autopsied Patients with Rheumatoid Arthritis in Japan. J Arthritis 2: 115. doi:10.4172/2167-7921.1000115

Page 2 of 4

(sepsis, respiratory infection, and others), malignancies (malignant lymphoma and others), cardiovascular disease, cerebrovascular disease, gastrointestinal disease (GI; gastrointestinal ulcers and others), renal disease, etc. A death caused by RA was defined as death from rheumatoid vasculitis or from articular complications such as atlantoaxial subluxation or cricoarytenoid arthritis. When a patient with ILD died from respiratory infection, the main cause of death was classified as ILD. Death due to failure of organs that were positive for amyloid deposition was classified as death from amyloidosis. The main pathological diagnosis was usually the main cause of death; however, in some cases where cause of death was not clearly written, the main cause of death was decided by two of the investigators (MK and CT) in discussion with other authors.

Differences in the main cause of death, age at death, gender, and AA-amyloidosis occurrence in autopsied patients with RA were compared between two periods, 2000-2004 and 1985-1989 [9]. We selected these time periods to see the effect of changes in drug therapy.

\section{Statistical analysis}

Patients' data (i.e., data from males, females, and all patients) are expressed as mean \pm SD. Differences in the frequency of death due to RA or its complications were compared between periods (2000-2004 and 1985-1989) and genders using the chi-square test. Differences in age at death were analyzed using a $t$-test. Differences were considered significant if the p value was less than 0.05 .

\section{Results}

During 2000-2004, there were a total of 126,437 autopsied patients. During the same period, a total of 4,958,000 Japanese died and therefore the autopsied patients in this study represented $2.5 \%$ of all deaths in this study period. The male-to-female ratio in autopsied patients was 1.84. A total of 1225 patients ( 408 males and 817 females) with RA were identified. RA was found in $0.50 \%$ and $1.84 \%$ of autopsied males and females, respectively. The adjusted male-to-female ratio of autopsied patients with RA was 3.7, considering the male-to-female ratio of all autopsies as 1.0. Mean age at death is shown for RA patients in Table 1A. Between 1985-1989 and 2000-2004, mean lifespan was extended for patients with RA (by 3.6 years in males and 4.5 years in female). Of note, and in contrast to observations in the general population, survival was significantly shorter in females with RA than males with RA. AAAmyloidosis was the third most frequent cause of death (11.3\%) in RA patients (2000-2004; $4.7 \%$ in males and $14.7 \%$ in females). Pathological deposition of AA-amyloid was shown in $26.3 \%$ of RA patients (20002004; $32.1 \%$ in females with RA and $14.7 \%$ in males with RA). The mean lifespan of patients with RA (2000-2004) either associated or not associated with AA-amyloid deposition was $67.3 \pm 9.8$ years and $70.1 \pm 9.5$ years, respectively. Having pathological AA-amyloidosis significantly shortened the lifespan of both genders with RA, even though the main cause of death was not necessarily AA-amyloidosis (Table 1B). The lifespan of patients who had AA-amyloid deposition without dying from AA-amyloidosis was shorter than that of patients who died from AA-amyloidosis.

Table 2 shows the causes of death in autopsied patients with RA

\begin{tabular}{|l|l|l|l|}
\hline Age at death & \multicolumn{3}{|l|}{ Autopsied cases with RA during } \\
\hline & $\begin{array}{l}1985-89 \\
(\mathrm{~N}=1246)\end{array}$ & $\begin{array}{l}2000-04 \\
(\mathrm{~N}=1225)\end{array}$ & Difference \\
\hline Males, mean $\pm \mathrm{SD}$, years & 66.5 & $70.1 \pm 9.3$ & 3.6 \\
\hline Females, mean $\pm \mathrm{SD}$, years & 64.6 & $69.1 \pm 9.8$ & 4.5 \\
\hline
\end{tabular}

Table 1A: Age at death in autopsied patients with RA in Japan.

\begin{tabular}{|l|l|l|l|l|c|}
\hline \multicolumn{7}{|c|}{ Age at death } \\
\hline & With amyloidosis & \multicolumn{1}{c|}{ Without amyloidosis } & p \\
\hline & No. & Mean \pm SD & No. & Mean \pm SD & \\
\hline Total RA & 322 & $67.3 \pm 9.8$ & 903 & $70.1 \pm 9.5$ & $<0.0001$ \\
\hline Male RA & 61 & $67.5 \pm 9.9$ & 347 & $70.5 \pm 9.2$ & 0.025 \\
\hline Female RA & 261 & $67.2 \pm 9.8$ & 556 & $70.0 \pm 9.7$ & 0.0002 \\
\hline
\end{tabular}

Table 1B: Age at death of autopsied RA patients with and without AA-amyloidosis.

\begin{tabular}{|c|c|c|c|c|c|c|}
\hline \multirow[t]{2}{*}{ Cause of death } & \multicolumn{2}{|c|}{$\begin{array}{c}\text { Cases during } \\
1985-89\end{array}$} & \multicolumn{2}{|c|}{$\begin{array}{c}\text { Cases during } \\
2000-04\end{array}$} & \multicolumn{2}{|c|}{ Current/previous } \\
\hline & No. & $(\%)$ & No. & $(\%)$ & OR & $\mathrm{P}$ \\
\hline Total & $1246^{*}$ & $(100.0)$ & 1225 & $(100.0)$ & & \\
\hline $\mathbf{R A}^{* *}$ & 173 & $(13.9)$ & 17 & (1.4) & 0.09 & $<0.0001$ \\
\hline Articular & 124 & $(10.0)$ & 4 & $(0.3)$ & 0.03 & $<0.0001$ \\
\hline Vasculitis & 49 & (3.9) & 13 & (1.1) & 0.26 & $<0.0001$ \\
\hline Amyloidosis & 156 & $(12.5)$ & 139 & $(11.3)$ & 0.89 & NS \\
\hline Respiratory & 218 & $(17.5)$ & 247 & $(20.2)$ & 1.19 & NS \\
\hline ILD & 166 & $(13.3)$ & 199 & $(16.2)$ & 1.26 & 0.04 \\
\hline Others & 52 & $(4.2)$ & 48 & (3.9) & 0.94 & NS \\
\hline Infection & 331 & $(26.6)$ & 402 & $(32.8)$ & 1.35 & 0.0007 \\
\hline Sepsis & 52 & $(4.2)$ & 93 & (7.6) & 1.89 & 0.0003 \\
\hline Respiratory & 204 & $(16.4)$ & 251 & $(20.5)$ & 1.31 & 0.008 \\
\hline Others & 75 & $(6.0)$ & 58 & $(4.7)$ & 0.75 & NS \\
\hline Lymphoma & - & - & 19 & (1.6) & - & - \\
\hline Others & - & - & 79 & (6.4) & - & - \\
\hline Cardiovascular & 104 & (8.3) & 128 & $(10.4)$ & 1.28 & 0.07 \\
\hline Cerebrovascular & 44 & (3.5) & 30 & $(2.4)$ & 0.69 & NS \\
\hline Digestive & 92 & (7.4) & 121 & $(9.9)$ & 1.54 & 0.003 \\
\hline Renal & 60 & $(4.8)$ & 19 & $(1.6)$ & 0.31 & $<0.0001$ \\
\hline Others & 73 & (5.9) & 24 & $(2.0)$ & 0.32 & $<0.0001$ \\
\hline
\end{tabular}

*In the earlier RA series, 105 autopsied patients died of multiple causes and the sum of all causes of death exceeded the total number of autopsy cases. ${ }^{* *}$ Death caused by RA itself (e.g., by articular lesions or rheumatoid vasculitis) RA: Rheumatoid Arthritis; ILD: Interstitial Lung Disease.

Table 2: Change between 1985-1989 and 2000-2004 in the cause of death in autopsied patients with rheumatoid arthritis in Japan.

during the two periods. During both periods, infection and respiratory diseases were, respectively, the first and second most common causes of death. AA-amyloidosis was the fourth and third most common cause of death in 1985-1989 and 2000-2004, respectively. Death from rheumatoid articular lesions and rheumatoid vasculitis decreased significantly during the 15 years between survey periods. On the other hand, death due to infection (especially sepsis and respiratory infection) and due to ILD increased significantly. Death due to amyloidosis decreased from $12.5 \%$ to $11.3 \%$ but not significantly. Cardiovascular death increased and cerebrovascular death decreased similarly in both the RA and general populations (data not shown).

Gender differences in the cause of death in RA patients during 2000-2004 are shown in Table 3. The leading cause of death in males and females was infection. Respiratory disease, especially ILD, was the second most common cause of death in males and the third most common cause of death in females. On the other hand, amyloidosis was second for females and sixth for males. Thus, ILD was prevalent among males, while amyloidosis was prevalent among females. The mean age at death caused by RA itself was significantly lower in females. Death due to respiratory infection tended to occur in older RA patients, while death due to cerebrovascular disease and AA-amyloidosis occurred in younger female RA patients.

\section{Discussion}

In this study, mortality in a nationwide group of RA patients 


\begin{tabular}{|c|c|c|c|c|c|c|c|c|}
\hline \multirow[b]{2}{*}{ Cause of death } & \multicolumn{3}{|c|}{ Males } & \multicolumn{3}{|c|}{ Females } & \multicolumn{2}{|c|}{ Gender } \\
\hline & No. & $(\%)$ & $\begin{array}{l}\text { Age at death } \\
\text { Mean } \pm \text { SD }\end{array}$ & No. & $(\%)$ & $\begin{array}{l}\text { Age at death } \\
\text { Mean } \pm \text { SD }\end{array}$ & OR & $\mathbf{P}$ \\
\hline RA* $^{*}$ & 4 & $(1.0)$ & $71.8 \pm 5.1$ & 13 & $(1.6)$ & $63.9 \pm 0.5^{\star *}$ & 0.61 & NS \\
\hline Amyloidosis & 19 & $(4.7)$ & $68.6 \pm 7.8$ & 120 & $(14.7)$ & $67.7 \pm 9.4$ & 0.28 & $<0.0001$ \\
\hline Respiratory & 128 & $(31.4)$ & $70.4 \pm 7.7$ & 119 & $(14.6)$ & $69.7 \pm 10.0$ & 2.68 & $<0.0001$ \\
\hline ILD & 109 & $(26.7)$ & $70.7 \pm 7.5$ & 90 & $(11.0)$ & $69.1 \pm 1.3$ & 2.94 & $<0.0001$ \\
\hline Infection & 130 & (31.9) & $69.5 \pm 10.8$ & 272 & (33.3) & $69.7 \pm 9.6$ & 0.93 & NS \\
\hline Sepsis & 21 & $(5.1)$ & $63.5 \pm 16.5$ & 72 & $(8.8)$ & $68.1 \pm 10.2$ & 0.56 & 0.022 \\
\hline Respiratory & 95 & $(23.3)$ & $71.4 \pm 8.8$ & 156 & $(19.1)$ & $70.9 \pm 9.5^{\star * *}$ & 1.28 & NS \\
\hline Malignancy & 33 & $(8.1)$ & $70.6 \pm 7.1$ & 65 & $(8.0)$ & $69.7 \pm 10.5$ & 1.01 & NS \\
\hline Lymphoma & 7 & $(1.7)$ & $71.0 \pm 3.9$ & 12 & $(1.5)$ & $68.7 \pm 9.1$ & 1.17 & NS \\
\hline Cardiovascular & 49 & $(12.0)$ & $71.0 \pm 3.9$ & 79 & $(9.7)$ & $70.5 \pm 9.4$ & 1.27 & NS \\
\hline Cerebrovascular & 4 & $(1.0)$ & $69.4 \pm 11.6$ & 26 & $(3.2)$ & $63.5 \pm 12.4^{* * * *}$ & 0.30 & 0.019 \\
\hline Digestive & 29 & $(7.1)$ & $66.5 \pm 13.7$ & 92 & (11.3) & $69.0 \pm 8.9$ & 0.60 & 0.022 \\
\hline Renal & 4 & $(1.0)$ & $78.8 \pm 4.6^{\star \star \star *}$ & 15 & $(1.8)$ & $67.5 \pm 8.4$ & 0.52 & NS \\
\hline Others & 8 & $(2.0)$ & $71.4 \pm 6.3$ & 16 & $(2.0)$ & $72.3 \pm 0.2$ & & \\
\hline Total & 408 & & $70.1 \pm 9.3$ & 817 & & & $69.1 \pm 9.7$ & \\
\hline
\end{tabular}

Mean age at death due to one disease was compared to mean age at death of all the rest by unpaired t-test in males and females.

${ }^{*}$ Death caused by RA itself like articular lesion or rheumatoid vasculitis.

${ }^{* *}: p<0.001,{ }^{* * *}: p<0.01,{ }^{* * *}: p<0.05$

Table 3: Gender difference in cause of death in autopsied patients with rheumatoid arthritis in Japan.

autopsied between 2000 and 2004 was assessed. Increased mortality, i.e., reduced survival, of RA patients has been shown by numerous studies. In previously reported studies from Japan, most of them have been limited to a single institution with small-scale cohort [4]. Most studies used death certificates to determine cause of death, however, some have pointed out the inaccuracy of death certificates [2,3,11]. Indeed, the frequency of death due to AA-amyloidosis largely varied between studies because of differences in the methods of sampling $[5,12]$. A variety of reports that analyzed autopsied fatalities reported amyloidosis as a cause of death in 5.8-12.5\% of cases [4,5]. Amyloidosis might be underestimated without pathological evaluation, and the cause of death due to amyloidosis could also be characterized as renal, gastrointestinal, or cardiac disease. Pathological evidence of disease at autopsy in addition to clinical diagnosis in hospitalized patients could yield more accurate information. Thus, one of the strengths of our study is that AA-amyloidosis was identified histologically in the RA population.

Our study showed age of death as 70.1 years for RA male and as 69.1 years for RA female during 2000-2004. The average life expectancy in the general population obtained from the Statistics Bureau of Japan in 2003 showed 78.1 years for males and 84.9 years for females. Thus, the mean lifespan appears to be much shorter in the RA population than in the general population. The extension of mean lifespan for autopsied patients with RA between 1985-1989 and 2000-2004 (3.6 years in males and 4.5 years in female) were greater than that of life expectancy in general population between 1987 and 2003 (2.7 years in males and 3.8 years in females). However, since autopsies are usually performed when disease is more serious or cause of death is unknown, our group of RA patients might be different from those in the rest of the RA population. In this regard, in our outpatient clinic-based RA cohort of more than 5,000 patients (the IORRA cohort) [13,14] followed up since 2000,134 RA patients died between 2000 and 2004 and mean age at death was 70.9 years in males and 69.5 years in females (personal communication, Nakajima A, 2005). Of particular note is that the age at death is almost identical between our autopsied group and IORRA cohort, although these studies were conducted during the same period in different patient populations and used completely different methods. The hazard ratio for mortality in Japanese RA in only one cohort study was 1.60 [3]. These data support the applicability of our conclusion to the general RA population rather than simply to our limited cohort.

The difference in causes of death between our RA patients and those in the general population might explain this reduction in survival. The three most common causes of death in the general Japanese population (2003) were malignancy (35.5\%), cardiovascular disease (20.1\%), and cerebrovascular disease (15.2\%), representing more than $70 \%$ of all death causes. On the other hand, for RA population, these three causes represented only $20.9 \%$ of all causes of death. Cardiovascular disease was a less frequent cause of death in RA patients in 2000-2004 (10.4\%) than in the general patient population (19.8\%). In our RA patients, death was more frequently due to infectious disease, respiratory disease, reactive AA-amyloidosis, and digestive diseases including gastrointestinal bleeding.

Analysis by gender revealed a significant increase in AA-amyloidosis as a cause of death in females. The age at death due to AA-amyloidosis in females was 67.7 years, which was lower than the age at death from other causes. Moreover, the age at death in females was significantly lower in RA associated with AA-amyloid deposition (67.2 years) than in RA without AA-amyloid deposition (70.0 years). AA-amyloid protein deposition in target organs is accelerated by an increased level of serum amyloid $A$ that is an acute phase protein similar to $C$ reactive protein [15]. Thus, AA-amyloidosis, as well as severe articular lesions and rheumatoid vasculitis, which are related to severe disease activity, explain in part the reduced survival of females with RA.

Comparison of causes of death in two autopsy series (one in 19851989 and one in 2000-2004) indicated death due to manifestations of severe RA (such as articular damage and vasculitis) and death due to renal diseases decreased significantly, while death due to infectious diseases and digestive diseases increased. Survival has improved more in RA patients than in the general population during this period. These improvements and changes in the causes of death probably reflect changes in drug therapy. During these 15 years, the incidence of severe RA has decreased and overall prognosis of RA has improved even though death from infection has increased. MTX was officially approved in 1999 in Japan and the first biologics (infliximab) was approved in 2003 in Japan. Therefore, the effect of MTX and infliximab 
Citation: Kitahama M, Okamoto H, Terai C, Koseki Y, Sakurai T, et al. (2013) Causes of Death in Autopsied Patients with Rheumatoid Arthritis in Japan. J Arthritis 2: 115. doi:10.4172/2167-7921.1000115

Page 4 of 4

on the prevention of AA amyloidosis might be appeared later than 2000-2004. In addition, approved dose of MTX was only up to $8 \mathrm{mg} /$ week until Feb. 2011 in Japan (then, again only up to $16 \mathrm{mg} /$ week, which is lower than Western dose). Thus, if we perform the same research 10 years later (for example, 2010-2014), AA amyloidosis might be reduced. The reasons for the increase in death due to infection appear to be multifactorial and include the introduction of more potent immunosuppressive drugs, higher average age of the patients, and a decrease in number of deaths for other reasons (such as RA itself and renal diseases). Although introduction of new drugs (anti-TNF biologics, leflunomide, and tacrolimus) is expected to further increase the life expectancy of RA patients, more attention should be paid to infectious disease as a cause of death. Cardiovascular disease has been recognized as a main cause of death among Caucasians with RA [16,17]. According to Wolf et al., [17] the rate of death due to infection is higher in the RA population than the general population in the US and rate of death due to malignancy is lower. The leading cause of death in both groups was cardiovascular disease ( $40.2 \%$ of deaths in the RA population and $40.5 \%$ of deaths in the general population), but because of premature deaths of RA patients, the Standardized Mortality Ratio (SMR) for cardiovascular disease in the RA population was 2.3. On the other side, cardiovascular disease accounted for only $10.4 \%$ of deaths in our autopsied RA population and $19.8 \%$ of deaths in the Japanese general population. It was the fourth most common cause of death in our autopsied population. Cardiovascular death is less prevalent in Japan, and earlier death from infection, ILD, or amyloidosis in Japanese patients with RA may reduce the prevalence of cardiovascular disease.

In summary, the causes of death in autopsied Japanese patients with RA have changed during the past 15 years. Death from severe RA has been decreasing but death from AA-amyloidosis has not changed, indicating that tighter control of inflammation is essential in the management of RA in Japan.

\section{Acknowledgments}

Supported by a grant provided by the Japanese Ministry of Health, Labour and Welfare (CT)

\section{References}

1. Cobb S, Anderson F, Bauer W (1953) Length of life and cause of death in rheumatoid arthritis. N Engl J Med 249: 553-556.

2. Maradit-Kremers H, Nicola PJ, Crowson CS, Ballman KV, Gabriel SE (2005)
Cardiovascular death in rheumatoid arthritis: a population-based study. Arthritis Rheum 52: 722-732

3. Hakoda M, Oiwa H, Kasagi F, Masunari N, Yamada M, et al. (2005) Mortality of rheumatoid arthritis in Japan: a longitudinal cohort study. Ann Rheum Dis 64: $1451-1455$.

4. Suzuki A, Ohosone Y, Obana M, Mita S, Matsuoka Y, et al. (1994) Cause of death in 81 autopsied patients with rheumatoid arthritis. J Rheumatol 21: 33-36.

5. Myllykangas-Luosujärvi R, Aho K, Kautiainen H, Hakala M (1999) Amyloidosis in a nationwide series of 1666 subjects with rheumatoid arthritis who died during 1989 in Finland. Rheumatology (Oxford) 38: 499-503.

6. Smolen JS, Aletaha D (2006) What should be our treatment goal in rheumatoid arthritis today? ClinExpRheumatol 24: S-7-13.

7. Bakker MF, Jacobs JW, Verstappen SM, Bijlsma JW (2007) Tight control in the treatment of rheumatoid arthritis: efficacy and feasibility. Ann Rheum Dis 66 Suppl 3: 56-60.

8. Choi HK, Hernán MA, Seeger JD, Robins JM, Wolfe F (2002) Methotrexate and mortality in patients with rheumatoid arthritis: a prospective study. Lancet 359: 1173-1177.

9. Toyoshima H, Kusaba T, Yamaguchi M (1993) [Cause of death in autopsied RA patients]. Ryumachi 33: 209-214.

10. Arnett FC, Edworthy SM, Bloch DA, McShane DJ, Fries JF, et al. (1988) The American Rheumatism Association 1987 revised criteria for the classification of rheumatoid arthritis. Arthritis Rheum 31: 315-324.

11. Laakso M, Isomäki $H$, Mutru O, Koota K (1986) Death certificate and mortality in rheumatoid arthritis. Scand J Rheumatol 15: 129-133.

12. Lachmann HJ, Goodman HJ, Gilbertson JA, Gallimore JR, Sabin CA, et al. (2007) Natural history and outcome in systemic AA amyloidosis. N Engl J Med 356: 2361-2371.

13. Matsuda Y, Singh G, Yamanaka H, Tanaka E, Urano W, et al. (2003) Validation of a Japanese version of the Stanford Health Assessment Questionnaire in 3,763 patients with rheumatoid arthritis. Arthritis Rheum 49: 784-788.

14. Yamanaka H, Tohma S (2006) Potential impact of observational cohort studies in Japan on rheumatoid arthritis research and practice. Mod Rheumatol 16 : 75-76

15. Merlini G, Bellotti V (2003) Molecular mechanisms of amyloidosis. N Engl J Med 349: 583-596.

16. Mutru O, Laakso M, Isomäki H, Koota K (1985) Ten year mortality and causes of death in patients with rheumatoid arthritis. Br Med J (Clin Res Ed) 290: 1797 1799.

17. Wolfe F, Mitchell DM, Sibley JT, Fries JF, Bloch DA, et al. (1994) The mortality of rheumatoid arthritis. Arthritis Rheum 37: 481-494. 